Letters A/H1N1 flu pandemic

\title{
Life threatening infections labelled swine flu
}

BMJ 2010; 340 doi: http://dx.doi.org/10.1136/bmj.c137 (Published 13 January 2010) Cite this as: BMJ 2010;340:c137

Catherine F Houlihan, specialty trainee in infectious diseases 1 , Sanjay Patel, registrar2, David A Price, consultant in infectious diseases 1 , Manoj Valappil, consultant virologist 3 , Uli Schwab, consultant in infectious diseases 1

${ }^{1}$ Department of Infectious Diseases and Tropical Diseases, Newcastle upon Tyne Hospitals NHS Trust, Newcastle upon Tyne NE1 4LP

${ }^{2}$ Department of Paediatric Infectious Diseases/Immunology, Newcastle upon Tyne Hospitals NHS Trust, Newcastle upon Tyne NE4 6BE

${ }^{3}$ North East England Regional Laboratory, Health Protection Agency, Newcastle upon Tyne NE4 6BE

catherine.houlihan@doctors.org.uk

Algorithms for remote diagnosis and issue of antiviral drugs are indispensable during a pandemic.1 Their application through the National Pandemic Flu Service to both high and low prevalence areas is, however, controversial, and the lack of specificity in the use of the algorithm has been highlighted by Payne et al and at November's meeting of the Federation of Infection Societies in Birmingham.2

Over six weeks (1 July 2009 to 15 August 2009) we reviewed cases of potentially life threatening conditions admitted to the Newcastle infection services in which diagnosis and management were delayed because of an initial, incorrect diagnosis of swine flu. During this time, rates of novel H1N1 swab positivity (22/336) suggested a local prevalence of $6.5 \%$ of patients presenting to hospital with a flu-like illness compared with $11.8 \%$ throughout England. 3

A label of swine flu resulted in an average diagnostic delay of three days in six adults and two children who were admitted with potentially life threatening infection requiring timely antimicrobials. They had instead meningococcal meningitis; severe (11\% parasitaemia) and mild $(0.2 \%)$ Plasmodium falciparum malaria complicated by renal failure; acute myeloblastic leukaemia presenting with febrile pancytopenia; Campylobacter gastroenteritis with renal failure; Haemophilus influenzae respiratory tract infection (bone marrow transplant recipient); complicated soft tissue infection; and a fatal Staphylococcus aureus bacteraemia with multiorgan failure.

Our results show that a concise history must cover travel, immunosuppression, and drug exposure comprehensively and that current local epidemiological data should influence the interpretation and application of algorithms. Algorithms do not replace comprehensive history taking, clinical acumen, laboratory support, and, above all, common sense.

\section{Notes}


Cite this as: $B M J$ 2010;340:c137

\section{Footnotes}

- Competing interests: None declared.

\section{References}

1. Maynard A, Bloor K. The economic impact of pandemic influenza. BMJ2009;339:b4888. (19 November.)

2. Payne R, Darton TC, Greig JM. Systematic telephone triage of possible "swine" influenza leads to potentially serious misdiagnosis of infectious diseases. J Infect 2009;59:371-2.

3. Health Protection Agency. Weekly national influenza report 20. August 2009 (week 34). 